\title{
Manganese Oxide Nanozymes Ameliorate Mechanical Allodynia in a Rat Model of Partial Sciatic Nerve-Transection Induced Neuropathic Pain
}

This article was published in the following Dove Press journal: International Journal of Nanomedicine

\author{
Yaswanth Kuthati (D) \\ Prabhakar Busa ${ }^{2}$ \\ Venkata Naga Goutham \\ Davuluri $^{3}$ \\ Chih Shung Wong \\ 'Department of Anesthesiology, Cathy \\ General Hospital, Taipei, Taiwan; \\ ${ }^{2}$ Department of Life Sciences, National \\ Dong Hwa University, Hualien, Taiwan; \\ ${ }^{3}$ Department of Microbiology and \\ Immunology, College of Medicine, \\ National Cheng Kung University, Tainan, \\ Taiwan
}

Background: Reactive oxygen species (ROS) induced oxidative stress is linked to numerous neurological diseases, including neuropathic pain. Natural ROS scavenging enzymes like superoxide dismutase (SOD) and catalase have been found to be efficient in alleviating neuropathic pain. However, their sensitivity towards extreme $\mathrm{pH}$ and a short half-life limit their efficacy in vivo. Manganese oxide nanoparticles (MONPs) are recently known to possess ROS scavenging properties. In this study, MONPs were examined for their therapeutic effect on neuropathic pain.

Methods: The MONPs were synthesized by a hydrothermal method. The synthesized MONPs were characterized by UV/Vis, TEM, SEM, FTIR, NTA and XRD. The biocompatibility of the nanoparticles is evaluated in neural cells using LDH assay. MONPs were evaluated for their antioxidant activity by DPPH assay. In addition, in vitro ROS scavenging properties were examined in bone marrow-derived macrophage (BMDM) cells using $2^{\prime}, 7^{\prime}-$ dichlorofluorescin diacetate (DCFDA) assay. To evaluate the in vivo efficacy of nanoparticles, neuropathic pain was induced in Wistar rats by partial sciatic nerve transection (PSNT). On post-transection days 14 to 18 , rats were intrathecally injected with MONPs and paw withdrawal threshold was measured. The spinal cords were collected and processed for Western blotting and histological analysis.

Results: The synthesized MONPs were biocompatible and showed effective antioxidant activity against DPPH free radical scavenging. Further, the nanoparticles scavenged ROS efficiently in vitro in BMDM and their intrathecal administration significantly reduced mechanical allodynia as well as the expression of cyclooxygenase-2 (COX-2), an important mediator of chronic and inflammatory pain in the spinal dorsal horns of PSNT rats.

Conclusion: As ROS play a significant role in neuropathic pain, we expect that MONPs could be a promising tool for the treatment of various inflammatory diseases and might also serve as a potential nanocarrier for the delivery of analgesics.

Keywords: allodynia, MONPs, reactive oxygen species and neuropathic pain

\section{Introduction}

Neuropathic pain (NP) is defined as the pain resulting from damage or injury to the somatosensory system. This type of pain is challenging to treat; common causes include chemotherapy, diabetes, ischemic disease, HIV, alcoholism, multiple sclerosis and surgery. ${ }^{1} \mathrm{NP}$ is majorly accompanied by symptoms such as allodynia (pain resulting from a normal non-painful stimulus such as a gentle touch due to amplified neuronal response) and hyperalgesia (increased pain sensitivity). The current treatment options for NP include pharmacology and intervention approaches. Although a variety
Correspondence: Chih Shung Wong Department of Anesthesiology, Cathay General Hospital, \#280, Renai Road, Section 4, Taipei, Taiwan

Tel +88622708212I Ext 3510

Emailw82556@gmail.com 
of strategies are used for the management of NP, very few have shown clinical success. ${ }^{1}$ In addition, insufficient pain relief, addiction, tolerance and severe side effects of analgesics are limitations that require innovative therapeutic interventions. The limited effectiveness of pharmacological interventions is due to the numerous etiologies of neuropathic pain.

Recent studies have revealed that reactive oxygen species (ROS) play a critical role in triggering, establishing, and maintaining $\mathrm{NP}^{2}$ The increased lipid content in the neural network leaves it very vulnerable to ROS. Earlier studies have suggested that oxidative stress in the spinal cord plays an important role in pain hypersensitivities induced by peripheral nerve damage and cutaneous neurogenic inflammation. ${ }^{3-5}$ Accumulation of ROS in the spinal cord is known to play a major role in the development of neuropathic pain by activation of several inflammatory mediators such which enhance the neuronal excitability. ${ }^{6-8}$ Specifically, ROS scavengers such as $N$-tert-butyl- $\alpha$-phenylnitrone, 4-hydroxy-2,2,6,6-tetramethylpiperidine 1-oxyl and vitamin E were shown to reverse mechanical allodynia. ${ }^{9-11}$ Though ROS is known to play a major role in establishing and maintaining NP, there is a shortage of effective clinical treatments that primarily target ROS system. Collectively, these studies suggest that ROS play an important role in central sensitization mechanism for mechanical allodynia and it is worth studying the effect of ROS scavengers in neuropathic pain treatment.

Antioxidants are the substances that can eliminate the fatal consequences from ROS. The natural antioxidants are primarily of three types: phytochemicals, vitamins, and enzymes. ${ }^{12}$ Though natural antioxidants are efficient in ROS scavenging, their high sensitivity towards environmental conditions such as temperature, $\mathrm{pH}$, low bioavailability and short half-life limits their usage in a clinical setting. In addition, natural antioxidants are hard to mass produce. ${ }^{13}$ Therefore, rigorous efforts have been made to develop artificial ROS scavengers to overcome the limitations of the natural antioxidants. ${ }^{14}$

In recent years, catalytic NPs bearing anti-oxidant like properties (termed as nano-antioxidants) have garnered significant attention. ${ }^{15}$ These nano-antioxidants have numerous advantages over natural anti-oxidants, such as the ease of mass production, high thermal and biological stability, multi-functionality, and tunable nature. A variety of inorganic nanoparticles with ROS scavenging properties have been discovered. ${ }^{16}$ For instance, cerium oxide $\left(\mathrm{CeO}_{2}\right)$ and yttrium oxide $\left(\mathrm{Y}_{2} \mathrm{O}_{3}\right)$ are known to mimic natural antioxidant enzymes like superoxide dismutase (SOD) and catalase (CAT). ${ }^{17}$ Both $\mathrm{CeO}_{2}$ and $\mathrm{Y}_{2} \mathrm{O}_{3}$ NPs are reported to possess neuroprotective properties and are known to have therapeutic qualities in the treatment of neurodegenerative diseases. ${ }^{17}$ Among the two, particularly $\mathrm{CeO}_{2}$ NPs are known to catalyze the same electron transfer reactions as glutathione, SOD and CAT by switching their mixed valance states $\mathrm{Ce}^{3+}$ and $\mathrm{Ce}^{4+}$ and scavenge the superoxide anions, hydrogen peroxide and peroxynitrite radicals. $^{18}$

Among other nano-antioxidants studied, MONPs have garnered significant attention, due to the numerous oxidation states of manganese (II, III, IV and VII), which make it possible to serve as a redox medium for ROS scavenging with multi-enzymatic functions. Recently, Singh et al have demonstrated that MONPs can protect the cells from oxidative damage to cellular components such as DNA, protein and lipids without the dependence on endogenous antioxidant machinery. ${ }^{19}$ However, till date, only a few Mn-based nano-oxidants have been developed for applications in vivo. Recently, MONPs are shown to bear ROS scavenging properties in vivo for hyperbilirubinemia, hepatic fibrosis and ear-inflammation. ${ }^{20-22}$ However, manganese oxide nano-antioxidants have not yet been studied for the treatment of neuropathic paint.

To fill this gap, herein we synthesized MONPs that possessed remarkable antioxidant properties. We showed that the MONPs not only exhibited excellent ROS removal capabilities in vitro in bone marrow-derived macrophages but also effectively alleviated neuropathic pain in vivo in Wistar rats through the suppression of COX-2 levels.

\section{Materials and Methods Materials}

Manganese (II) acetate tetrahydrate was purchased from Chem-Impex international, hydrogen peroxide $\left(\mathrm{H}_{2} \mathrm{O}_{2}, 30 \%\right)$ was obtained from Merck-Millipore, 2',7'-dichlorofluorescin diacetate (DCFHDA) and 2,2-Diphenyl-1-picrylhydrazyl (DPPH) are purchased from Sigma Aldrich. All chemical reagents were used as received without any further purification. All aqueous solutions were prepared using deionized water.

\section{Animals}

The protocols used in our study were reviewed and approved by the Animal Care and Use Committee of the National Defense Medical Center and comply with the 
guidelines given by National Institute of Health Guide for the Care and Use of Laboratory Animals. Male Wistar at 7 weeks of age (BioLASCO Taiwan Co., Ltd., Taiwan) were housed ( 2 per cage) with soft bedding material on a $12 \mathrm{hr}$ night/day cycle with unrestricted access to food and water. All efforts were taken to minimize the animal number and suffering.

\section{Instrumentation}

Transmission electron microscopy (TEM) images were obtained on a Tecnai G2 TF 20 Super Twin electron microscope and the images were acquired by using Digital Micrograph (Gatan, Inc.). UV-vis spectra were recorded using a Genequant-1300 series spectrophotometer. The size determination of MONPs was performed with a nanoparticle tracking (NTA) (Nanosight NS 300, Malvern, PA, USA) system. The size of the particles was evaluated in a buffer solution (pH7.4). The crystalline structure was analyzed using XRD (PAnalytical X'pert $\mathrm{PRO})$ with $\mathrm{Cu}-\mathrm{K} \alpha$ radiation (=1.541À). FT-IR spectra were recorded using the Bruker Alpha spectrometer with a $\mathrm{KBr}$ pellet. Approximately $1 \mathrm{mg}$ of a sample was mixed with $300 \mathrm{mg}$ of dried $\mathrm{KBr}$ and then pressed.

\section{Synthesis of MONPs}

The MONPs were synthesized using the following procedure. $^{20,23}$ Firstly, $1.225 \mathrm{~g}$ of manganese (II) acetate tetrahydrate was dissolved in $60 \mathrm{~mL}$ anhydrous ethanol and stirred for 30 mins. Then the mixture was transferred into a teflon-lined stainless-steel container and autoclaved overnight at $120{ }^{\circ} \mathrm{C}$ temperature. The obtained dark brown nanoparticle precipitants are repeatedly washed with deionized water and stored in ethanol at room temperature for further use.

\section{LDH Assay}

Cytotoxicity of MONPs was evaluated using an LDH cytotoxicity detection kit (Roche applied sciences). This assay measures the leakage of cytoplasm enzyme lactate dehydrogenase (LDH) up on cellular damage. CNS-1 cells obtained from ATCC are $1 \times 10^{4}$ cells are cultured in 96 plates in $10 \%$ DMEM for 1 day and were treated with increasing concentrations of MONPs $(10,20,40,60$, and $100 \mu \mathrm{g} / \mathrm{mL}$ ). After $48 \mathrm{hrs}$, supernatant was collected and incubated with reaction mixture. The LDH catalyzed conversion leads to the reduction of tetrazolium salt to formosan, which can be read at $490 \mathrm{~nm}$. TitonX-100 was used as a positive control and DMEM is used as a negative control. Any significant increase in LDH levels would indicate cell membrane damage or death.

\section{DPPH Radical Scavenging Assay}

The DPPH radical scavenging activity of manganese nanoparticles was determined according to the previously reported procedure. ${ }^{24}$ Different concentrations $(5000 \mu \mathrm{g}$ / $\mathrm{mL}-40 \mu \mathrm{g} / \mathrm{mL}$ ) of nanoparticles are prepared by serial dilution in ethanol. Nanoparticle suspension of $0.1 \mathrm{~mL}$ is added to $1 \mathrm{~mL}$ of $0.1 \mathrm{mM}$ freshly prepared DPPH solution in ethanol. The reaction mixture was shaken vigorously for 10 mins and then nanoparticles were centrifuged for and the supernatants absorbance was recorded at $517 \mathrm{~nm}$ in a UV spectrophotometer. The radical scavenging efficacy was determined as a measure of decrease in absorbance by using the following formula.

$$
\begin{aligned}
& \text { DPPH scavenging } \operatorname{activity}(\%) \\
& \quad=\left[\left(\mathrm{Abs}_{\text {control }}-\mathrm{Abs}_{\text {sample }}\right) /\left(\mathrm{Abs}_{\text {control }}\right)\right] \times 100
\end{aligned}
$$

where Abs control is the absorbance of DPPH + methanol and $\mathrm{Abs}$ sample is the absorbance of DPPH + sample (MONPs).

\section{Bone Marrow-Derived Cell Culture and ROS Evaluation}

Murine bone marrow-derived macrophages (BMDMs) were produced by gently flushing bone marrow cells from femurs and tibias of $6-10$-week old BALB/c mice. These collected cells were cultured for a week in RPMI media (Gibco) with $10 \%$ FBS and $10 \mathrm{ng} / \mathrm{mL}$ recombinant mouse M-CSF (Peprotech, Rocky Hill, NJ, USA). To evaluate the ROS scavenging efficacy of MONPs the cultured BMDM cells $4 \times 10^{5}$ cells are seeded in six-well plates and divided into four groups. The first group is left without any treatment (Control), the second group is treated with MONPs $(20 \mu \mathrm{g})$ and the third group is treated with PAM3CSK $4(5 \mu \mathrm{g} / \mathrm{mL})$. The fourth group is pre-incubated for 30 mins with MONPs $(20 \mu \mathrm{g})$ followed by the addition of PAM3CSK4 with additional incubation for $12 \mathrm{hrs}$. Finally, DCFHDA is added to all four groups and readings are obtained at FL-1 channel using a flow cytometer.

\section{Establishment of the Neuropathic Pain Animal Model and Intrathecal Catheterization}

Partial sciatic nerve transection is performed according to previously reported protocols. ${ }^{25}$ The left sciatic nerve was gently exposed till mid-thigh level, and a prolene 7-0 ligature 
was inserted through the midpoint of the nerve just cranially to the branch running to the musculus biceps femoris, half of the nerve is transected in the ventrocranial direction up to the ligature. In the sham group, the sciatic nerve was gently exposed, and wound was closed with sutures. From the surgery day to day 14 the paw withdrawal thresholds are measured in sham-operated rats and PSNT rats to mechanical stimulus. Rats presenting any sign of motor deficit were excluded. Fourteen days post PSNT intrathecal catheter is implanted under $2 \%$ to $2.5 \%$ isoflurane anesthesia. ${ }^{26,27}$ Intrathecal catheter was constructed similar to our previous reports. ${ }^{28}$ At first, the rat's cisternal membrane is carefully pierced to insert a polyethylene catheter (PE 10 tubing, $8.0 \mathrm{~cm}$ ) and threaded caudally to reach the lumbar enlargement of the spinal cord. The rostral end of the catheter was exteriorized at the top of the head, and the wound was closed with sutures. The nanoparticles are suspended in saline before administration. To test the anti-allodynic efficacy, $50 \mu \mathrm{g}$ of MONPs in $10 \mu \mathrm{L}$ saline are intrathecally administered through a syringe. After nanoparticle administration, the catheters were flushed with $15 \mu \mathrm{L}$ saline and 30 mins post-injection the animals are randomly assigned to undergo paw withdrawal threshold test. The same procedure is repeated on days 16,17 and 18 post-PSNT. Same procedures of spinal aspiration are performed on sham group rats with the same volume of saline injection without MONPS.

\section{Behavior Test for Tactile Allodynia}

The sensitivity of the plantar region of the left paw was measured using an automatic Dynamic Plantar Aesthesiometer (Ugo Basile, Comerio, Italy). Rats were separately placed in a plastic enclosure ( $25 \mathrm{~cm}$ long $\times 10 \mathrm{~cm}$ wide $\times 14 \mathrm{~cm}$ high) with a wiremeshed base and acclimatized for 20 mins before each session of testing and then a paw withdrawal response was initiated by gradually increasing the force using a blunt-end metal filament $(0.5 \mathrm{~mm})$ pointed to the mid-plantar surface of the hind paw. The force was initially below the detection threshold, and then was gradually amplified from 1 to $50 \mathrm{~g}$ in $1 \mathrm{~g}$ steps over 25 seconds, then maintained at $50 \mathrm{~g}$ for another 10 seconds. The paw withdrawal threshold was taken as the force required to initiate hind paw reflex and was recorded as the mean of three measurements at 2-min time intervals.

\section{Behavior Test for Thermal Hyperalgesia}

In this test, thermal hypersensitivity was examined using a Hargreaves radiant heat apparatus (7371; Ugo Basile, Comerio, Italy; infrared setting 80 ). The animals were placed in a plastic enclosure $(23 \mathrm{~cm}$ long $\times 18 \mathrm{~cm}$ wide $\times 14 \mathrm{~cm}$ high $)$ with plantar test apparatus at the base. Initially, the animals are acclimatized for 15 mins before behavioral testing. The apparatus is equipped with a movable noxious heat source placed directly below the plantar surface. Upon activation, the apparatus generates a continuous infrared heat stimulus pointed towards the plantar surface of the hind paw, and a distinctive paw withdrawal reflex was provoked, upon which the automated timer records the withdrawal response time. The animals were tested alternately, with a 5-min interval, and three measurements of latency were averaged for each hind paw in individual test sessions. The baseline threshold was between 8 and 10 seconds in naive rats, and a 22 -second cutoff time was set to prevent paw damage.

\section{Antinociception Test}

Tail flick latency was tested using a hot water immersion bath $\left(52 \pm 0.5^{\circ} \mathrm{C}\right)$. The baseline latency was approximately $2 \pm 0.38$ seconds for naïve rats, and 10 seconds was set as the cut-off time. Rats were placed in plastic restrainers for the tail-flick test after the treatment of nanoparticles and the latency was checked at 30,60 and 90 seconds after treatment with the nanoparticles.

\section{Spinal Cord Preparation and Western Blotting Analysis}

After the behavioral testing, all the rats were euthanized under anesthesia with isoflurane (Abbott Laboratories Ltd., Queenborough, Kent, United Kingdom), and the left dorsal quadrant portion of the lumbar spinal cord enlargement was detached and stored at $-80^{\circ} \mathrm{C}$. The spinal cord samples were homogenized in ice-cold 1X RIPA lysis buffer (Temecula, CA) using an ultrasonic cell disruptor (Misonix, Inc. USA), then the homogenate was centrifuged at 13,000 RPM for 30 mins at $4^{\circ} \mathrm{C}$. The supernatant was collected, and its protein concentration was determined using the Bradford protein assay. The proteins were denatured by heating at $90^{\circ} \mathrm{C}$ for 10 mins in an equal volume of reducing sample buffer and subjected to separation using $12 \%$ SDS-PAGE followed by protein transfer on to a PVDF membrane (Pall, Ann Arbor, MI, USA) and blocked using 5\% skimmed milk in TBST (0.05\% Tween 20 in TBS). Primary antibodies against COX2 (ab52237; Abcam, Massachusetts, USA) were incubated at $4{ }^{\circ} \mathrm{C}$ overnight followed by the wash with TBST. Further, incubated with HRP-conjugated goat anti-rabbit (Leadgene Biomedical Cat\# 20202) antibody for another $3 \mathrm{~h}$ and detected using an Enhanced Chemiluminescence Western Blotting Kit (Advansta, Menlo Park, CA, USA). 


\section{Hematoxylin and Eosin Staining}

After the treatment for 18 days, the histological analysis was performed by examining the morphological changes induced by MONPs in the dorsal horn of rats. Dorsal Horns were embedded in TissueTek OCT compound (Bayer). The OCT sections were cut $(5 \mu \mathrm{m})$, mounted on Superfrost Plus slides (Thermo Fisher Scientific, Rockford, IL), dried for $1 \mathrm{hr}$ at room temperature, stained for analysis using hematoxylin and eosin ( $\mathrm{H}$ and $\mathrm{E})$ staining kit (abcam, ab245880). The sections were examined under fluorescent microscope and images were obtained.

\section{Statistical Analysis}

Unpaired data were evaluated using Student's $t$-test to compare two mean values, and one-way analysis of variance (ANOVA) followed by Tukey's multiple-comparison test was used to compare more than two mean values. All data were expressed as mean \pm standard deviation. In all analyses, a P-value $<0.01$ was considered statistically significant.

\section{Results and Discussion Synthesis and Characterization of MONPs}

Hydrothermal method is used for the synthesis of MONPs. ${ }^{23} \mathrm{Uv}$-Vis, TEM, SEM and PXRD were employed to study the morphological characteristics of the nanomaterials. Figure S1 shows the UV-Vis spectra of as assynthesized MONPs. Scanning at the wavelength $\left(\lambda_{\max }\right)$ between 200 and 800 revealed the existence of $\mathrm{Mn}_{3} \mathrm{O}_{4}$ pattern that matched with JCPDS card (\# 024-0734). As shown in Figure 1A, the synthesized nanoparticles have a uniform polyhedral shape with an average size of $10 \mathrm{~nm}$. Figure S2 shows the data obtained by NTA for particle size distribution of the MONPs. Most particles are within a size range of 10-300 nm, which is higher than the mean size of TEM due to the covalent bonding between the nanoparticles. The SEM images show that NPs have polyhedral to spherical shape with slight aggregation (Figure 1B). The powder XRD patterns (Figure 1C) showed that synthesized nanoparticles were crystalline. The obtained diffraction peaks matched well to the standard hausmannite-pattern (JCPDS: 24-0734) with no impurities, indicating the high purity of nanoparticles. To characterize the chemical structure, Fourier-transform infrared (FT-IR) spectroscopy was performed (Figure 1D) from 4000 to $400 \mathrm{~cm}^{-1}$. The spectrum displays the representative absorption bands of $v \mathrm{Mn}-$
$O$ vibrations at 615 and $496 \mathrm{~cm}^{-1}$ along with a weak band at $1098 \mathrm{~cm}^{-1}$, which can be ascribed to $\mathrm{vMn}-\mathrm{O}-\mathrm{H}$ vibration. Finally, a broadband at $3400 \mathrm{~cm}^{-1}$ and another weak band at $1623 \mathrm{~cm}^{-1}$ were characteristic water peaks.

\section{In vitro Innate Cytotoxicity Evaluation}

The Cns-1 neural cells are treated with a series of increasing concentrations of MONPs $(10,20,40,60$, and $100 \mu \mathrm{g} / \mathrm{mL})$ and LDH release was quantified, with DMEM treated cells as a negative control and Tritox-100 treated cells as positive control (Figure 2). At all concentrations, the nanoparticles showed excellent cell viability. These results are consistent with previous investigations where oral dose of MONPs at $5000 \mathrm{mg} / \mathrm{kg}$ body weight did not affect the liver function in rats. $^{21}$

\section{Antioxidant Activity of MONPs}

For antioxidant properties of MONPs were evaluated in vitro by using 2.2-diphenyl1-picrylhydrazyl (DPPH) free radical. The hydrogen atom or electron donation capability of the MONPs was measured from the decolorization of a purplecoloured ethanolic DPPH solution. ${ }^{29}$ The DPPH and lipid peroxyl radicals are known to have comparable structures and the reaction percentage of DPPH and antioxidants give a good estimate for scavenging activities with lipid peroxyl radicals. Various lipid peroxidation inhibitors are known to alleviate mechanical sensitivity in neuropathic pain animal models. ${ }^{30}$ The freshly prepared DPPH solution exhibited a deep purple color with a sharp peak at $517 \mathrm{~nm}$ Figure 3A. As shown in Figure 3B, the anti-oxidant properties of nanoparticles increased with the increase in nanoparticle concentration showing a maximum of $60 \%$ at $5 \mathrm{mg} / \mathrm{mL}$. These results showed that MONPs have strong antioxidant characteristics, and this activity could occur due to numerous oxidation states displayed by MONPs.

\section{MONPs Scavenge ROS in Bone Marrow-Derived Macrophages}

Of the various leukocytes present in the spinal cord after neuronal injury, macrophages predominate. Suppression of macrophage activation at the spinal level is shown to effectively alleviate neuropathic pain in various studies. ${ }^{31,32}$ Through the release of inflammatory mediators and enzymes involved in host defense, macrophages can damage neurons and glia. The ROS scavenging properties of MONPs are studied in bone marrow-derived macrophage cells (BMDM). The ROS generation is initiated in BMDM cells 

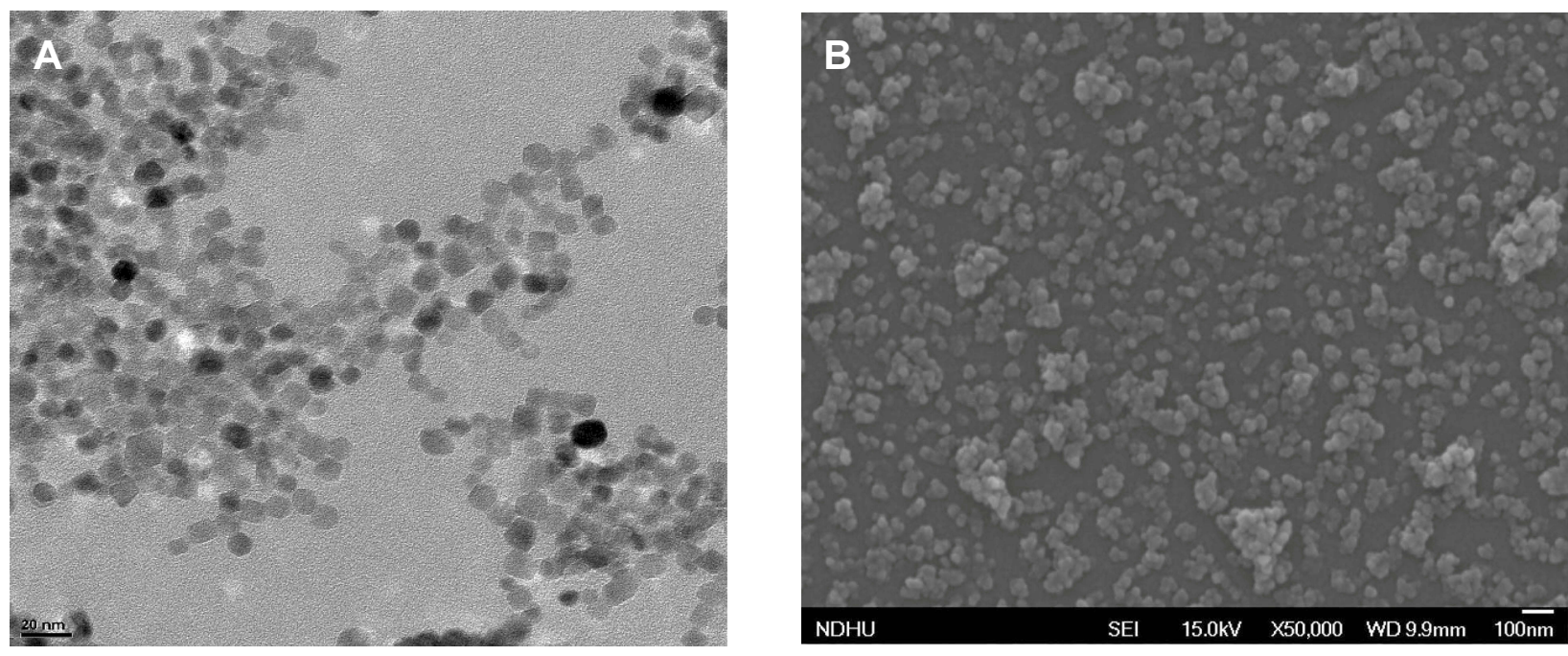

C

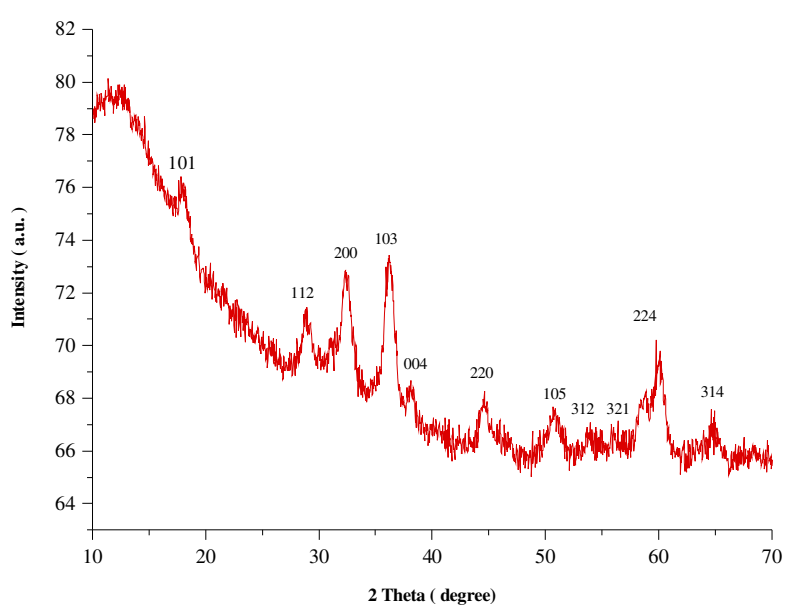

D

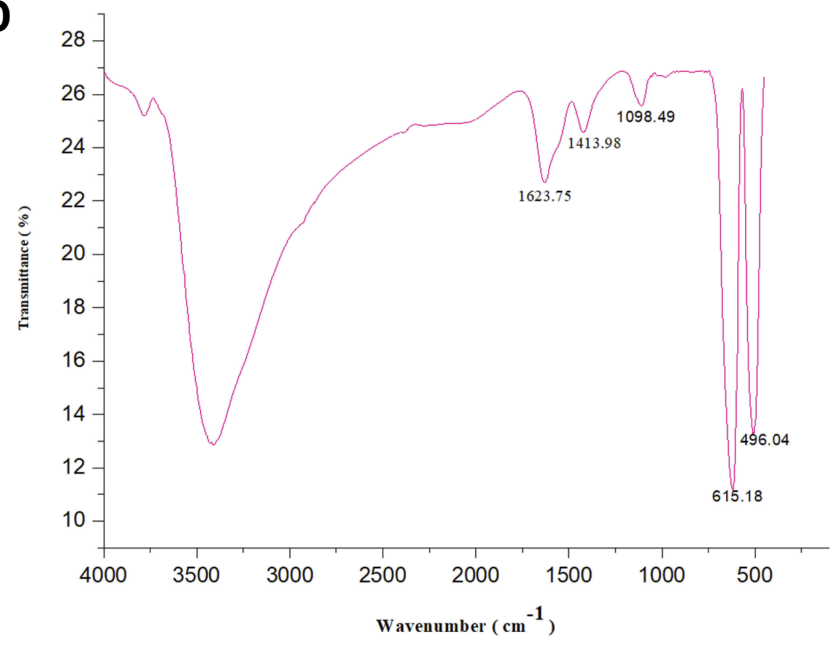

Figure I (A) Transmission electron microscopy micrograph of MONPs. (B) Scanning electron micrograph of MONPs. (C) X-ray powder diffraction patterns of MONPs and (D) Fourier-transform infrared spectra of MONPs.

Abbreviation: MONPs, manganese oxide nanoparticles.

by treating with Toll-like receptor-2 (TLR2) ligand PAM3CSK 4 and the ROS generated is quantified by treating with redox-sensitive dye DCFDA. ${ }^{33,34}$ Toll receptors are key initiators in mediating innate immune response and are activated upon various conditions ranging from pathogen encounter to nerve injury. ${ }^{35}$ These receptors are also known to play a key role in the activation of microglial cells, inflammation and the development of NP by triggering a signaling cascade that induces the production of reactive oxygen and nitrogen species. ${ }^{36,37}$ Significant elevation of cellular ROS $(\mathrm{P}<0.05)$ is observed in BMDM treated with PAM3CSK4 as compared to untreated controls Mock and MONPs (Figure 4). Pre-treating BMDM 30 mins with MONPs significantly inhibited the elevation of ROS levels on treatment with PAM3CSK4, indicating the ROS scavenging capabilities in PAM3CSK4 treated cells thereby maintaining a redox-environment comparable to that of mock and cells treated only with MONPs (Figure 4). These results indicate that ROS rapidly accumulates in macrophages upon treatment with PAM3CSK4, while pretreatment with MONPs can scavenge ROS, and levels remain close to that observed for untreated control.

\section{Intrathecal Injection of MONPs Attenuates Mechanical Allodynia but Not Thermal Hyperalgesia in PSNT Rats}

To examine whether MONPs could attenuate mechanical allodynia, we chose partial sciatic nerve transection (PSNT), a well-known model for assessing neuropathic pain behavior in rats. Various animal models have been 


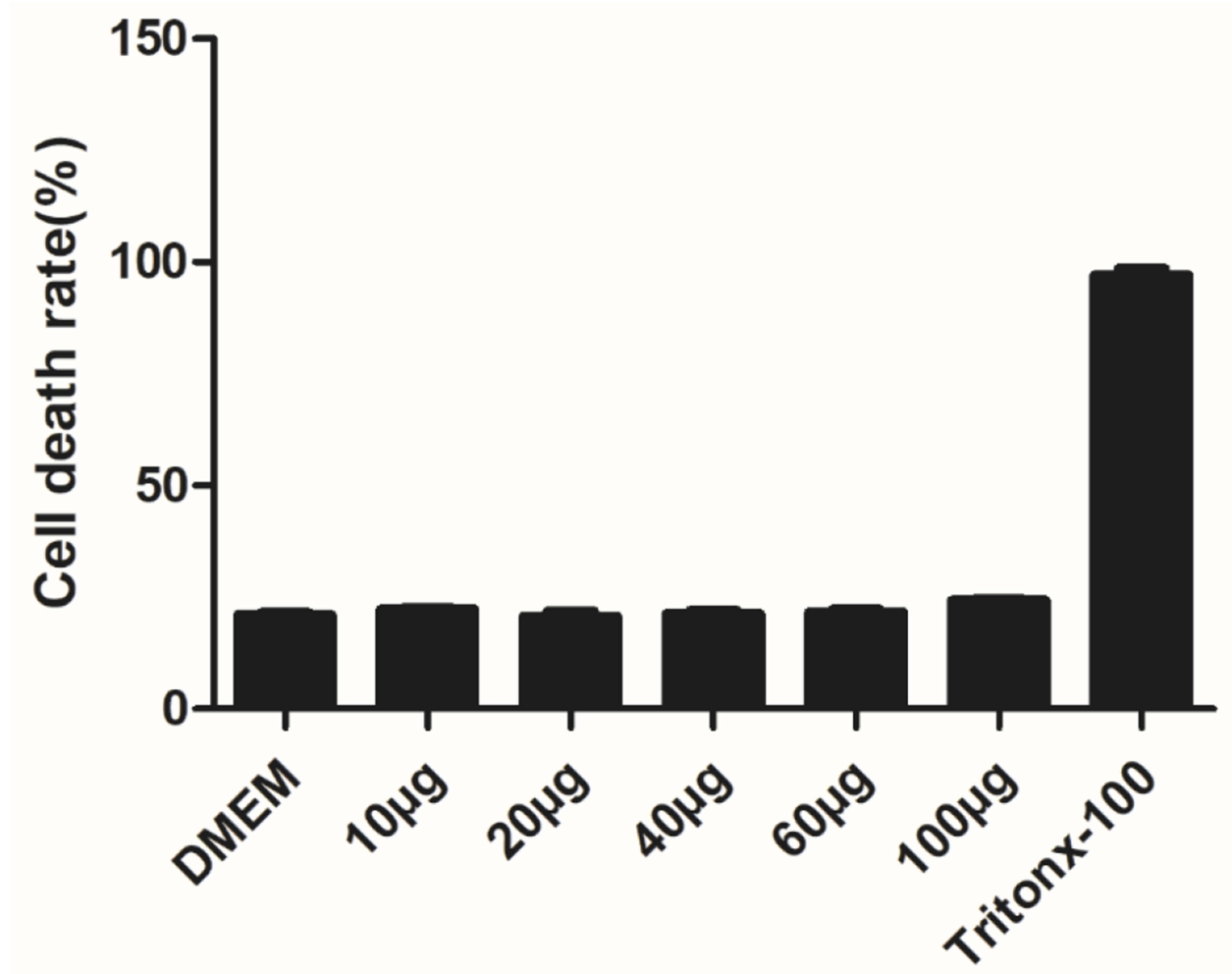

Figure 2 Effect of MONPs on LDH leakage in CnsI neuronal cells. Cytotoxicity was determined by LDH release after $24 \mathrm{~h}$ of exposure to MONPs (I0-100 $\mu \mathrm{g} / \mathrm{mL})$. Data are mean $\pm S D$. Results were calculated as percentage of the positive control (Triton X-I00-lysed CnsI) $(n=3)$.

Abbreviations: MONPs, manganese oxide nanoparticles; LDH, lactate dehydrogenase.
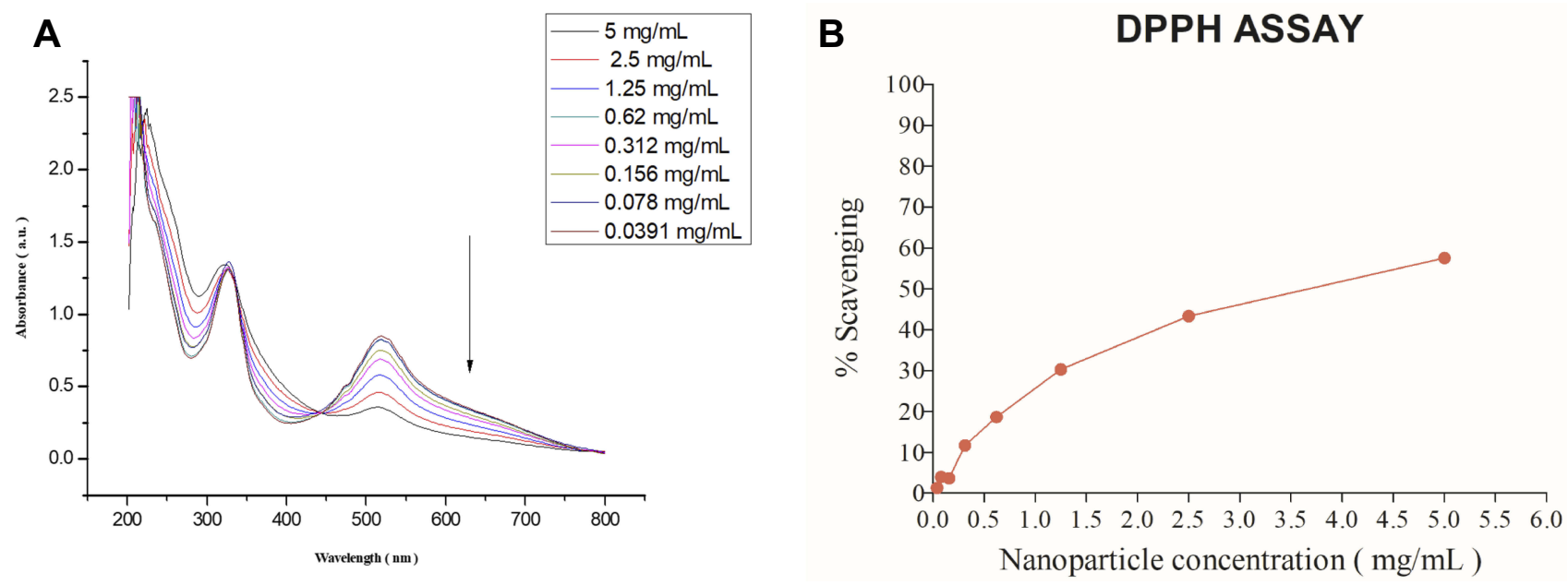

Figure 3 Absorption spectra of DPPH and oxidation of DPPH radicals. (A) As DPPH radicals are scavenged by the MONPs, there is a decrease in absorbance at $517 \mathrm{~nm}$ which is monitored by ultraviolet-visible spectroscopy (B) DPPH free radical scavenging percentage at different concentrations of MONPs.

Abbreviations: MONPs, manganese oxide nanoparticles; DPPH, 2,2-diphenyl-2-picrylhydrazyl hydrate.

developed to study neuropathic pain. Among them, sciatic nerve ligation (SNL) and chronic constriction injury (CCI) are well-known models. However, SNL model is susceptible to infections and motor deficit. ${ }^{25,38}$ Whereas the differences in pain perception depend on ligature snugness and differential immune responses due to the usage of ligatures is a key limitation for $\mathrm{CCI} .{ }^{25,38}$ As shown in Figure 5, we used a partial sciatic nerve transection (PSNT) that is known to have more advantages than the above reported models since a pure nerve injury is induced without the involvement of epineurial inflammatory component due to foreign material. ${ }^{25}$ Prior to PSNT surgery, the mechanical 

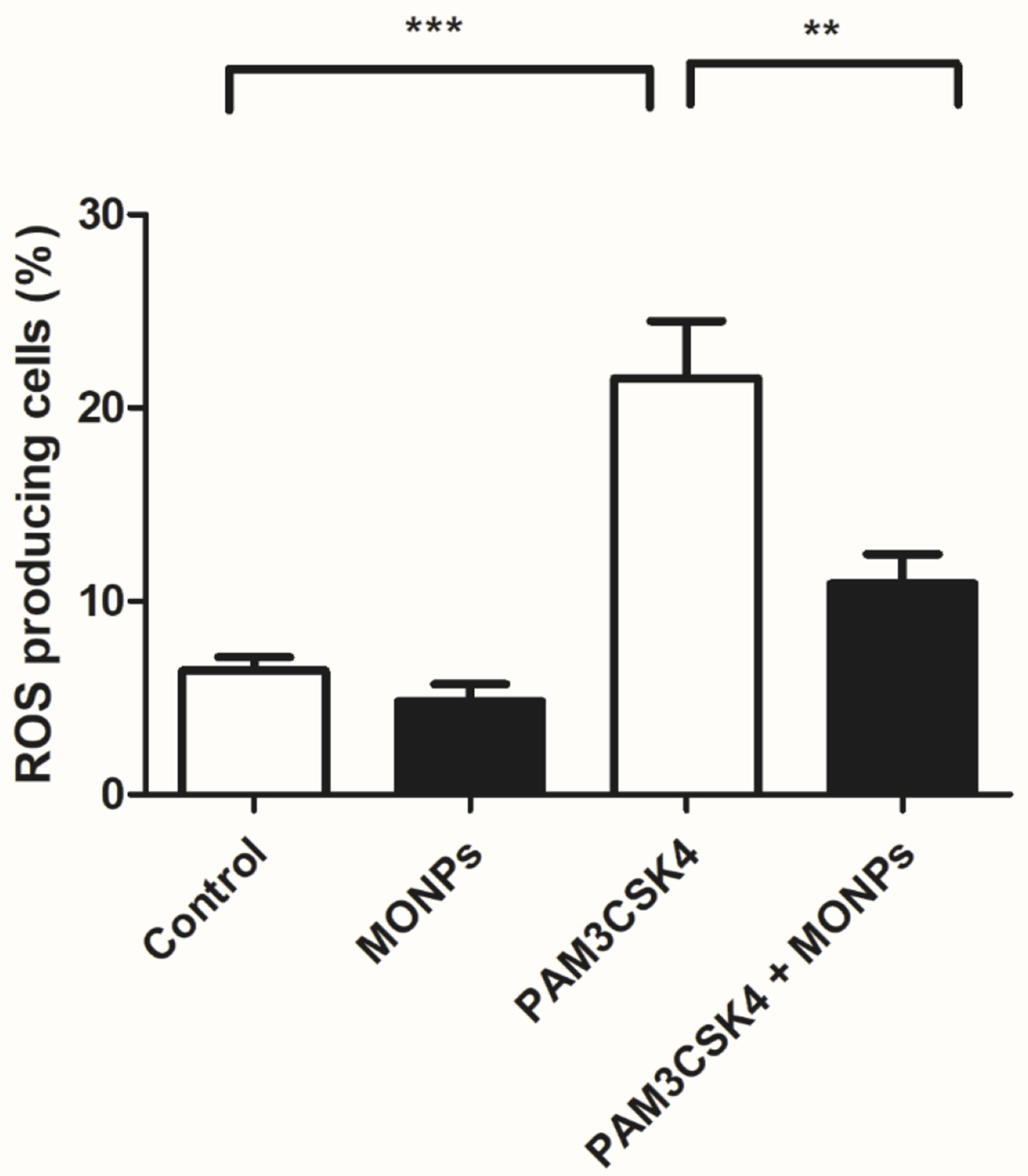

Figure 4 MONPs reduce ROS production induced by TLRI/2 agonist PAM3CSK4. The bone-derived macrophages are either untreated (Blank) or treated with MONPs (20 $\mu \mathrm{g})$ or stimulated with triacylated bacterial lipopeptide PAM3CSK $4(5 \mu \mathrm{g} / \mathrm{mL})$ or pre-incubated MONPs for 30 mins followed by the addition of PAM3CSK4 (5 $\mu \mathrm{g} / \mathrm{mL})$. The cells were further incubated for 12 hrs and readings were noted at FL-I channel using a flow cytometer after the addition of DCFHDA. ROS generation was measured in triplicate. The data shown are the mean \pm SD values of 3 individual experiments. ${ }^{* *} \mathrm{p}<0.0 \mathrm{I}$ versus untreated control, $* * * p<0.00 \mathrm{I}$ versus untreated control.

Abbreviations: MONPs, manganese oxide nanoparticles; PAM3CSK4, PAM3CSK4, Pam3CysSerLys4; TLR I/2, Toll-like receptor 1/2; DCFHDA, 2',7'-dichlorofluorescin diacetate; ROS, reactive oxygen species.

hypersensitivity of rats is determined by Dynamic Plantar Aesthesiometer. The rats with only those passing a predefined baseline $(>35 \mathrm{~g}$ ) were selected for PSNT surgery. Following PSNT, rats were assessed for mechanical hypersensitivity from day 1 to 14 post operation. A decrease in the mechanical threshold was observed in the left paw of all the rats that underwent PSNT from day 1 (Figure 6). Whereas animals the sham group did not show any mechanical hypersensitivity. To evaluate whether MONPs could attenuate mechanical hypersensitivity, MONPs were delivered by intrathecal injection using a $10 \mu \mathrm{L}$ 26-S gauge syringe needle (Hamilton, USA) into the pre-inserted intrathecal catheters of PSNT rats from days 14 to 18 post PSNT. Although the mechanical allodynia of rats treated with saline sustained until day 18 , rats treated with MONPs showed clear reduction in hypersensitivity from the initial treatment of nanoparticles on day 14 to the last day (day 18). Taken together, our results suggest that intrathecal administration of MONPs significantly improves PSNT-induced mechanical allodynia.

In comparison, the Hargreaves test showed no significant increase in thermal pain threshold after MONP administration (Figure S3). In general, patients with neuropathic pain show various kinds of abnormal sensations, including hyperalgesia (abnormal heightened sensitivity to noxious pain) and allodynia (central pain sensitization due to 
Neuropathic pain induction by partial sciatic nerve transection

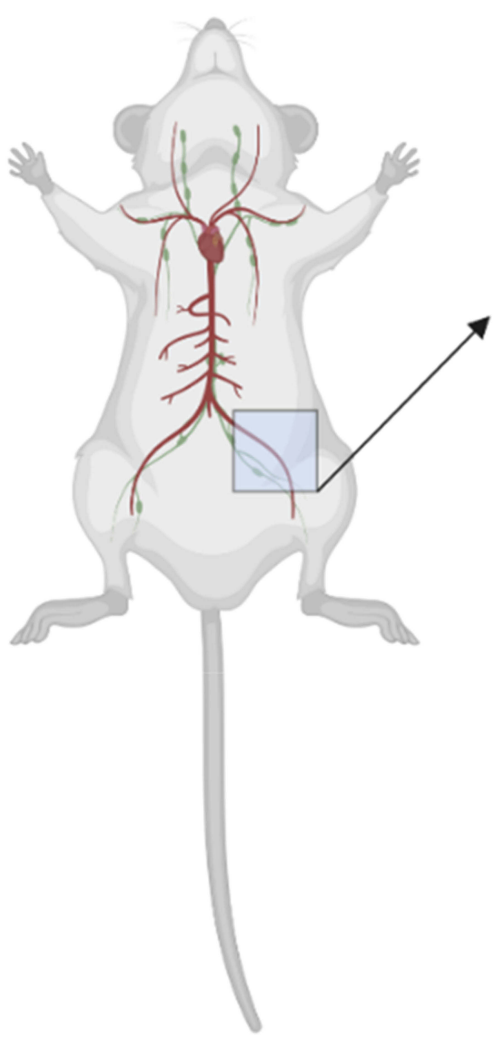

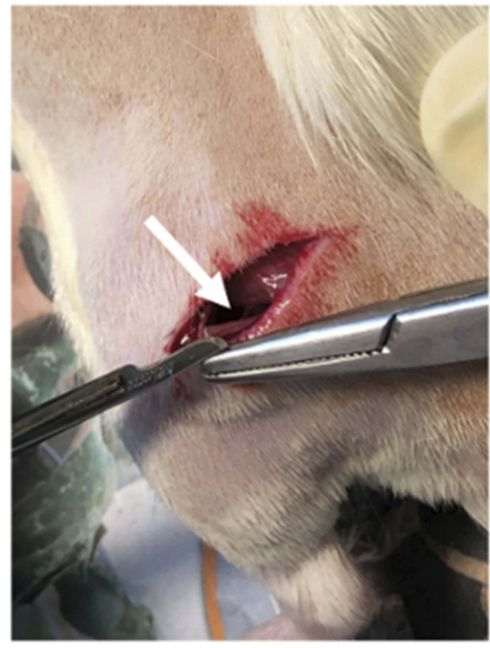

ROS elevation

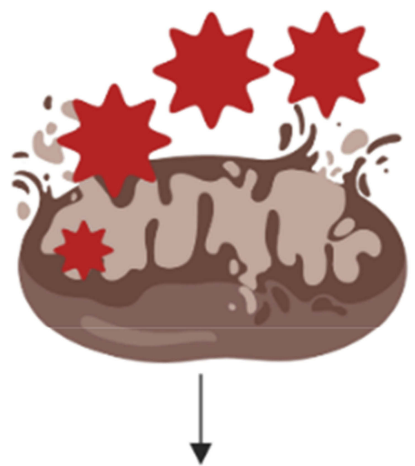

COX-2 expression

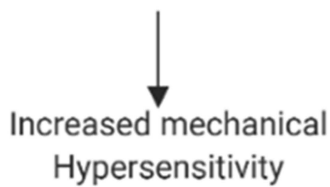

i.t. Injection

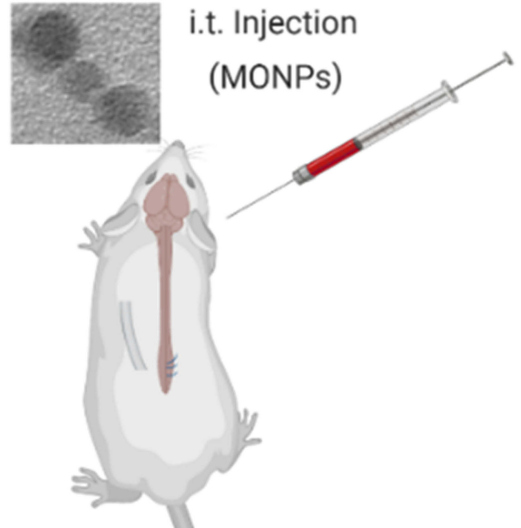

Free radical

neutralization

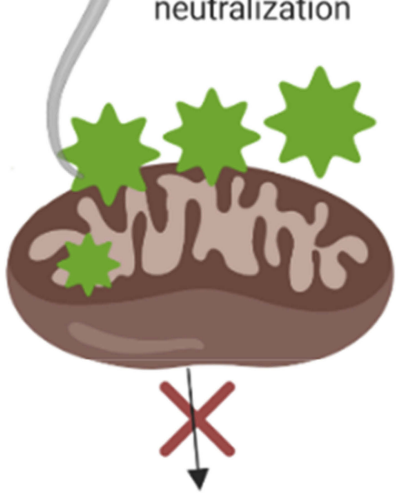

cox-2 inhibition

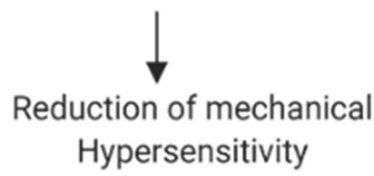

Figure 5 Experimental scheme for this study. Neuropathic pain was induced by partial sciatic nerve ligation in Wistar rats. MONPs were then administered intrathecally 14 days post nerve transection. Pain tested by using Dynamic Plantar Aesthesiometer was significantly attenuated in rats injected with MONPs compared with the salineinjected group. Scheme created with BioRender imaging software.

increased neuronal response). These abnormalities can occur either singly or in combination. This result highlights the fact that different mechanisms trigger the sensory disorders in neuropathic pain. This concept is supported by previous studies showing that different receptor systems are implicated in thermal hyperalgesia and mechanically evoked pain-related behavior. ${ }^{39-42}$ According to our current understanding, nitric oxide $\left(\mathrm{NO}^{\circ}\right)$ and superoxide $\left(\mathrm{O}_{2}{ }^{-}\right)$ reactive oxygen species have independent pain pathways wherein, nitric oxide-induced hyperalgesia was not affected by removing superoxide and superoxide-induced hyperalgesia was not affected by inhibiting nitric oxide. ${ }^{43}$ Some recent evidence shows that nerve transection induces the over-expression of $\mathrm{NO}^{\circ}$ contributing to hyperalgesia and is reversed by nNOS inhibitors. ${ }^{44,45}$ MONPs are reported to selectively scavenge superoxide $\left(\mathrm{O}_{2}{ }^{-}\right)$species but not nitric oxide (NO) ${ }^{\circ}$ species. ${ }^{46}$ This could have resulted in attenuation of mechanical allodynia without the attenuation of thermal hyperalgesia. We next investigated whether MONPs administration has any efficacy against nociceptive pain though tail flick test and we found no efficacy against nociceptive pain as expected (Figure S4).

\section{Cyclooxygenase 2 Protein Analysis}

COX-2 is a highly inducible protein which responds to inflammation and is often considered a target protein by various anti-inflammatory agents. Research evidence indicates that nerve injury and peripheral inflammation can spike 


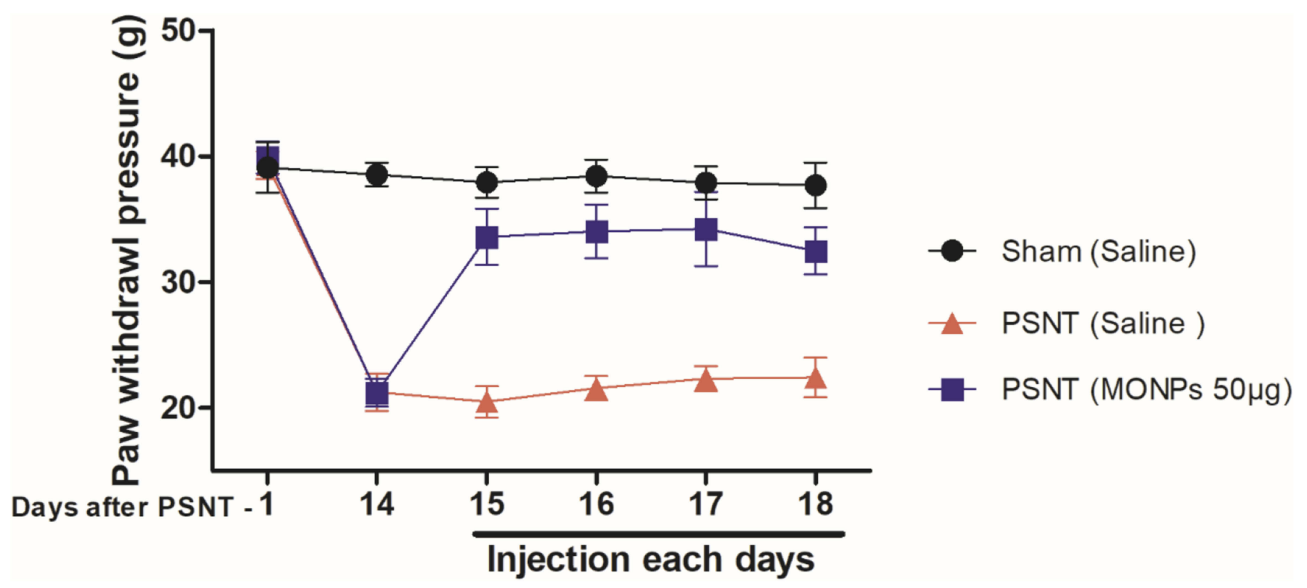

Figure 6 Time-course of the change in paw withdrawal threshold to mechanical stimulus in partial sciatic nerve transection (PSNT) rats and the effect of MONPs on mechanical paw withdrawal threshold in Sham and PSNT rats. Differences between paw withdrawal threshold to tactile stimuli in rats with sham surgery (N=6) and PSNT were assessed preoperatively on the day of surgery (indicated as day $-\mathrm{I}$ on the $\mathrm{x}$-axis) and on day 14 after surgery. After PSNT, significant tactile sensitivity developed on day 14 . The effect of intrathecal MONPs ( 50 $\mu \mathrm{g}) \mathrm{NPs}$ on the antihyperalgesic effect was measured for 60 mins after saline or nanoparticle injection from days 14 to 18 after PSNT. p<0.01.

Abbreviations: PSNT, partial sciatic nerve transection; MONPs, manganese oxide nanoparticles.

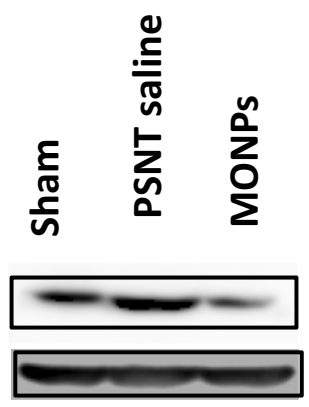

Figure 7 Effect of MONPs intrathecal single injection on COX-2 protein expression in spinal cord of sham surgery and PSNT animals through Western blotting analysis. $\boldsymbol{\beta}$-Actin is the loading protein control.

Abbreviations: MONPs, manganese oxide nanoparticles; COX-2, cyclooxygenase-2; PSNT, partial sciatic nerve transection.

the COX-2 mRNA levels and protein production. ${ }^{47,48}$ A chain of events relating ROS generation with $N F-\kappa B$ activation and consequent $\mathrm{COX}-2$ protein production is well documented in literature. ${ }^{24,49,50}$ Intrathecal administration of COX-2 inhibitors was shown to prevent nerve injuryinduced mechanical allodynia. ${ }^{51-53} \mathrm{~A}$ wide range of nonsteroidal anti-inflammatory drugs are known to induce analgesic activities primarily through the inhibition of COX-1 and COX-2 levels. ${ }^{54}$ Some studied reported that enhanced COX-2 production leads to the release of spinal prostaglandins, which can produce increased neuronal excitability in the spinal cord (central sensitization). ${ }^{55}$ Research evidence also shows that anti-oxidants can suppress the levels of COX-2 and alleviate inflammatory response. ${ }^{56,57}$ COX-2 expression after nervous injury has been known to play an important major role in peripheral and central sensitization, by altering the excitability of the nociceptor peripheral terminal. However. ${ }^{58,59}$

To determine the role of COX-2 on PSNT-induced neuropathic pain, we first examined whether COX-2 expression levels are altered by nerve transection. As shown in Figure 7, PSNT increased the expression of COX-2 protein levels in the spinal cord and treatment with MONPs attenuated the concentration of COX-2 compared to PSNT vehicle-treated rats (Figure 7), demonstrating the anti-oxidant efficacy of the manganese oxide nanomaterials. These data indicate that MONPs relieve PSNT-induced neuropathic pain in rats by the downregulation of proinflammatory gene COX-2 in the spinal dorsal horn.

\section{Hematoxylin and Eosin Staining}

Activation of glial cells including microglial cells, astrocytes, and oligodendrocytes is known to play a key role in the pathology of neuroinflammation and neuropathic pain. In the present study, histological analysis (Figure S5) revealed that there is a significant spike in the number of astrocytes and oligodendrocytes in the dorsal horn of rats that underwent PSNT in comparison with sham rats. Treatment with MONPs reduced the activation of astrocytes, and oligodendrocytes. In addition, MONP treatment did not reveal any signs of inflammation in the dorsal horn, and no significant changes occurred in immune subpopulations when compared with those in controls. Our results are consistent with previous investigations where oral dose of MONPs even at 
a high dose of $5000 \mathrm{mg} / \mathrm{kg}$ body weight did not affect the liver function nor hepatic morphological in rats. ${ }^{21}$

\section{Conclusion}

In summary, we have designed an efficient antioxidant defense platform for ROS elimination in partial sciatic nerve transection-induced neuropathic pain by using MONPs as intelligent ROS scavengers. A series of important experiments were performed in this study to enhance our comprehensive understanding of the antioxidative effects of MONPs. In vitro experiments using bonederived macrophage cells revealed that the MONPs were efficient in scavenging intracellular ROS. Due to their extraordinary antioxidant properties in vitro, neuropathic pain was induced in a rat model using partial sciatic nerve transection to evaluate the ROS scavenging properties in vivo. The MONPs efficiently relieved the PSNTinduced mechanical allodynia in Wistar rats through the suppression of inflammation-associated enzyme COX-2. Taking together, our present study not only provided a detailed insight in understanding the anti-oxidant properties of MONPs in neuropathic pain but also provided a promising platform for the development of metal nanoparticles as redox-active nanozymes for the treatment of inflammatory disorders.

\section{Future Prospects}

The present work can be extended further by incorporating anti-nociceptive drugs like morphine that are used for long-term chronic pain management. The ROS scavenging properties of manganese nanomaterials might minimize the morphine-tolerance development in long-term pain management as the development of morphine antinociceptive tolerance is prevented by substances with antioxidant properties. Moreover, extensive work is required to understand the peripheral effects of MONPs for their application in pain medicine.

\section{Acknowledgments}

We thank Ministry of Science and Technology, Taiwan and Cathay General Hospital for the grant support. (MOST: 106-2314-B-281-003-MY3;CMRI: CGH-MR-A10810). Thanks to Vaikar Navakanth Rao (Institute of Pharmacology and Toxicology, Tzu Chi University) for his helpful assistance with $\mathrm{H}$ and $\mathrm{E}$ staining.

\section{Disclosure}

The authors report no conflicts of interest in this work.

\section{References}

1. Murnion BP. Neuropathic pain: current definition and review of drug treatment. Aust Prescr. 2018;41(3):60-63. doi:10.18773/ austprescr.2018.022

2. Carrasco C, Naziroğlu M, Rodríguez AB, Pariente JA. Neuropathic pain: delving into the oxidative origin and the possible implication of transient receptor potential channels. Front Physiol. 2018;9:95. doi:10.3389/fphys.2018.00095

3. Park E-S, Gao X, Chung JM, Chung K. Levels of mitochondrial reactive oxygen species increase in rat neuropathic spinal dorsal horn neurons. Neurosci Lett. 2006;391(3):108-111. doi:10.1016/j.neulet.2005.08.055

4. Lee I, Kim HK, Kim JH, Chung K, Chung JM. The role of reactive oxygen species in capsaicin-induced mechanical hyperalgesia and in the activities of dorsal horn neurons. Pain. 2007;133(1-3):9-17. doi:10.1016/j.pain.2007.01.035

5. Schwartz ES, Lee I, Chung K, Chung JM. Oxidative stress in the spinal cord is an important contributor in capsaicin-induced mechanical secondary hyperalgesia in mice. Pain. 2008;138(3):514-524. doi:10.1016/j.pain.2008.01.029

6. Onodera Y, Teramura T, Takehara T, Shigi K, Fukuda K. Reactive oxygen species induce Cox-2 expression via TAK1 activation in synovial fibroblast cells. FEBS Open Bio. 2015;5:492-501. doi:10.1016/j.fob.2015.06.001

7. Moini Zanjani T, Ameli H, Labibi F, Sedaghat K, Sabetkasaei M. The attenuation of pain behavior and serum COX-2 concentration by curcumin in a rat model of neuropathic pain. Korean $J$ Pain. 2014;27(3):246-252. doi:10.3344/kjp.2014.27.3.246

8. Salvemini D, Little JW, Doyle T, Neumann WL. Roles of reactive oxygen and nitrogen species in pain. Free Radic Biol Med. 2011;51 (5):951-966. doi:10.1016/j.freeradbiomed.2011.01.026

9. Zhou Y-Q, Liu D-Q, Chen S-P, et al. Reactive oxygen species scavengers ameliorate mechanical allodynia in a rat model of cancer-induced bone pain. Redox Biol. 2018;14:391-397. doi:10.1016/j.redox.2017.10.011

10. Kim MJ, Hong BH, Zhang EJ, Ko YK, Lee WH. Antinociceptive effects of intraperitoneal and intrathecal vitamin $\mathrm{E}$ in the rat formalin test. Korean J Pain. 2012;25(4):238-244. doi:10.3344/kjp.2012.25.4.238

11. Carvalho LF, Silva AMF, Carvalho AA. The use of antioxidant agents for chemotherapy-induced peripheral neuropathy treatment in animal models. Clin Exp Pharmacol Physiol. 2017;44(10):971-979. doi:10.1111/1440-1681.12803

12. Neha K, Haider MR, Pathak A, Yar MS. Medicinal prospects of antioxidants: A review. Eur J Med Chem. 2019;178:687-704. doi:10.1016/j.ejmech.2019.06.010

13. Breslow R. Biomimetic chemistry and artificial enzymes: catalysis by design. Acc Chem Res. 1995;28(3):146-153. doi:10.1021/ar00051a008

14. Day BJ. Catalase and glutathione peroxidase mimics. Biochem Pharmacol. 2009;77(3):285-296. doi:10.1016/j.bcp.2008.09.029

15. Valgimigli L, Baschieri A, Amorati R. Antioxidant activity of nanomaterials. J Mater Chem B. 2018;6(14):2036-2051. doi:10.1039/ C8TB00107C

16. Sandhir R, Yadav A, Sunkaria A, Singhal N. Nano-antioxidants: an emerging strategy for intervention against neurodegenerative conditions. Neurochem Int. 2015;89:209-226. doi:10.1016/j.neuint.2015.08.011

17. Hosseini A, Sharifi AM, Abdollahi M, et al. Cerium and yttrium oxide nanoparticles against lead-induced oxidative stress and apoptosis in rat hippocampus. Biol Trace Elem Res. 2015;164(1):80-89. doi:10.1007/s12011-014-0197-z

18. Charbgoo F, Ahmad MB, Darroudi M. Cerium oxide nanoparticles: green synthesis and biological applications. Int $J$ Nanomed. 2017;12:1401-1413. doi:10.2147/IJN 
19. Singh N, Savanur MA, Srivastava S, D'Silva P, Mugesh G. A manganese oxide nanozyme prevents the oxidative damage of biomolecules without affecting the endogenous antioxidant system. Nanoscale. 2019;11(9):3855-3863. doi:10.1039/C8NR09397K

20. Yao J, Cheng Y, Zhou M, et al. ROS scavenging Mn3O4 nanozymes for in vivo anti-inflammation. Chem Sci. 2018;9(11):2927-2933. doi:10.1039/C7SC05476A

21. Adhikari A, Polley N, Darbar S, Bagchi D, Pal SK. Citrate functionalized $\mathrm{Mn}(3) \mathrm{O}(4)$ in nanotherapy of hepatic fibrosis by oral administration. Future Sci OA. 2016;2(4):FSO146-FSO146. doi:10.4155/fsoa-20160029

22. Polley N, Saha S, Adhikari A, et al. Safe and symptomatic medicinal use of surface-functionalized $\mathrm{Mn} 3 \mathrm{O} 4$ nanoparticles for hyperbilirubinemia treatment in mice. Nanomedicine (Lond). 2015;10 (15):2349-2363. doi: $10.2217 / \mathrm{nnm} .15 .83$

23. Li X, Zhou L, Gao J, Miao H, Zhang H, Xu J. Synthesis of Mn3O4 nanoparticles and their catalytic applications in hydrocarbon oxidation. Powder Technol. 2009;190(3):324-326. doi:10.1016/j. powtec.2008.08.010

24. Adderley SR, Fitzgerald DJ. Oxidative damage of cardiomyocytes is limited by extracellular regulated kinases $1 / 2$-mediated induction of cyclooxygenase-2. J Biol Chem. 1999;274(8):5038-5046. doi:10.1074/ jbc.274.8.5038

25. Lindenlaub T, Sommer C. Partial sciatic nerve transection as a model of neuropathic pain: a qualitative and quantitative neuropathological study. Pain. 2000;89(1):97-106. doi:10.1016/S0304-3959(00)00354-7

26. Yaksh TL, Rudy TA. Chronic catheterization of the spinal subarachnoid space. Physiol Behav. 1976;17(6):1031-1036. doi:10.1016/ 0031-9384(76)90029-9

27. Tai YH, Wang YH, Tsai RY, et al. Amitriptyline preserves morphine's antinociceptive effect by regulating the glutamate transporter GLAST and GLT-1 trafficking and excitatory amino acids concentration in morphine-tolerant rats. Pain. 2007;129(3):343-354. doi:10.1016/j. pain.2007.01.031

28. Tai YH, Wang YH, Wang JJ, Tao PL, Tung CS, Wong CS. Amitriptyline suppresses neuroinflammation and up-regulates glutamate transporters in morphine-tolerant rats. Pain. 2006;124(1-2):77-86. doi:10.1016/j. pain.2006.03.018

29. Mishra K, Ojha H, Chaudhury NK. Estimation of antiradical properties of antioxidants using DPPH assay: a critical review and results. Food Chem. 2012;130(4):1036-1043. doi:10.1016/j.foodchem.2011.07.127

30. Hassler SN, Johnson KM, Hulsebosch CE. Reactive oxygen species and lipid peroxidation inhibitors reduce mechanical sensitivity in a chronic neuropathic pain model of spinal cord injury in rats. J Neurochem. 2014;131(4):413-417. doi:10.1111/jnc.2014.131.issue-4

31. Popovich PG, Guan Z, Wei P, Huitinga I, van Rooijen N, Stokes BT. Depletion of hematogenous macrophages promotes partial hindlimb recovery and neuroanatomical repair after experimental spinal cord injury. Exp Neurol. 1999;158(2):351-365. doi:10.1006/exnr.1999.7118

32. Takeura N, Nakajima H, Watanabe S, Honjoh K, Takahashi A, Matsumine A. Role of macrophages and activated microglia in neuropathic pain associated with chronic progressive spinal cord compression. Sci Rep. 2019;9(1):15656. doi:10.1038/s41598-019-52234-1

33. Li Y, Deng SL, Lian ZX, Yu K. Roles of toll-like receptors in nitroxidative stress in mammals. Cells. 2019;8:6. doi:10.3390/cells8020137

34. Aranda A, Sequedo L, Tolosa L, et al. Dichloro-dihydro-fluorescein diacetate (DCFH-DA) assay: a quantitative method for oxidative stress assessment of nanoparticle-treated cells. Toxicol in Vitro. 2013;27(2):954-963. doi:10.1016/j.tiv.2013.01.016

35. Kim D, Lee S, Lee SJ. Toll-like receptors in peripheral nerve injury and neuropathic pain. Curr Top Microbiol Immunol. 2009;336:169-186. doi:10.1007/978-3-642-00549-7_10
36. Shi XQ, Zekki H, Zhang J. The role of TLR2 in nerve injury-induced neuropathic pain is essentially mediated through macrophages in peripheral inflammatory response. Glia. 2011;59(2):231-241. doi:10.1002/glia.v59.2

37. Li Y, Deng S-L, Lian Z-X, Yu K. Roles of toll-like receptors in nitroxidative stress in mammals. Cells. 2019;8(6):576. doi:10.3390/ cells8060576

38. Challa SR. Surgical animal models of neuropathic pain: pros and cons. Int $J$ Neurosci. 2015;125(3):170-174. doi:10.3109/ 00207454.2014.922559

39. Tal M, Bennett GJ. Neuropathic pain sensations are differentially sensitive to dextrorphan. Neuroreport. 1994;5(12):1438-1440. doi:10.1097/00001756-199407000-00008

40. Roh D-H, Kim H-W, Yoon SY, et al. Intrathecal injection of the §1Receptor antagonist BD1047 blocks both mechanical allodynia and increases in spinal NR1 expression during the induction phase of rodent neuropathic pain. Anesthesiology. 2008;109(5):879-889. doi:10.1097/ALN.0b013e3181895a83

41. Moriyama M, Kitamura A, Ikezaki H, et al. Systemic ATP infusion improves spontaneous pain and tactile allodynia, but not tactile hypesthesia, in patients with postherpetic neuralgia. $J$ Anesth. 2004;18(3):177-180. doi:10.1007/s00540-004-0240-x

42. Somers DL, Clemente FR. High-frequency transcutaneous electrical nerve stimulation alters thermal but not mechanical allodynia following chronic constriction injury of the rat sciatic nerve. Arch Phys Med Rehabil. 1998;79(11):1370-1376. doi:10.1016/S0003-9993(98)90230-1

43. Kim HY, Wang J, Lu Y, Chung JM, Chung K. Superoxide signaling in pain is independent of nitric oxide signaling. Neuroreport. 2009;20 (16):1424-1428. doi:10.1097/WNR.0b013e328330f68b

44. Chacur M, Matos RJ, Alves AS, et al. Participation of neuronal nitric oxide synthase in experimental neuropathic pain induced by sciatic nerve transection. Braz J Med Biol Res. 2010;43(4):367-376. doi:10.1590/S0100-879X2010007500019

45. Kumar SM, Porterfield DM, Muller KJ, Smith PJS, Sahley CL. nerve injury induces a rapid efflux of nitric oxide (NO) detected with a novel NO microsensor. $J$ Neurosci. 2001;21(1):215. doi:10.1523/ JNEUROSCI.21-01-00215.2001

46. Singh N, Geethika M, Eswarappa SM, Mugesh G. Manganese-based nanozymes: multienzyme redox activity and effect on the nitric oxide produced by endothelial nitric oxide synthase. Chemistry. 2018;24 (33):8393-8403. doi:10.1002/chem.201800770

47. Beiche F, Scheuerer S, Brune K, Geisslinger G, Goppelt-Struebe M. Up-regulation of cyclooxygenase-2 mRNA in the rat spinal cord following peripheral inflammation. FEBS Lett. 1996;390 (2):165-169. doi:10.1016/0014-5793(96)00604-7

48. Hay CH, Trevethick MA, Wheeldon A, Bowers JS, de Belleroche JS. The potential role of spinal cord cyclooxygenase- 2 in the development of Freund's complete adjuvant-induced changes in hyperalgesia and allodynia. Neuroscience. 1997;78(3):843-850. doi:10.1016/S0306-4522(96)00598-2

49. Cosentino F, Eto M, De Paolis P, et al. High glucose causes upregulation of cyclooxygenase- 2 and alters prostanoid profile in human endothelial cells: role of protein kinase $\mathrm{C}$ and reactive oxygen species. Circulation. 2003;107(7):1017-1023. doi:10.1161/ 01.CIR.0000051367.92927.07

50. Kiritoshi S, Nishikawa T, Sonoda K, et al. Reactive oxygen species from mitochondria induce cyclooxygenase-2 gene expression in human mesangial cells: potential role in diabetic nephropathy. Diabetes. 2003;52(10):2570-2577. doi:10.2337/diabetes.52.10.2570

51. Yaksh TL, Dirig DM, Conway CM, Svensson C, Luo ZD, Isakson PC. The acute antihyperalgesic action of nonsteroidal, anti-inflammatory drugs and release of spinal prostaglandin E2 is mediated by the inhibition of constitutive spinal cyclooxygenase- 2 (COX-2) but not COX-1. J Neurosci. 2001;21(16):5847-5853. doi:10.1523/JNEUROSCI.21-16-05847.2001 
52. Lui PW, Lee CH. Preemptive effects of intrathecal cyclooxygenase inhibitor or nitric oxide synthase inhibitor on thermal hypersensitivity following peripheral nerve injury. Life Sci. 2004;75(21):2527-2538. doi:10.1016/j.lfs.2004.04.033

53. Lee JY, Choi HY, Park CS, et al. Inhibition of COX-2 alleviates lumbar spinal stenosis-induced chronic mechanical allodynia in rats. Int Immunopharmacol. 2019;75:105738. doi:10.1016/j.intimp.2019.105738

54. Brune K, Patrignani P. New insights into the use of currently available non-steroidal anti-inflammatory drugs. J Pain Res. 2015;8:105-118. doi:10.2147/JPR.S75160

55. Samad TA, Moore KA, Sapirstein A, et al. Interleukin-1betamediated induction of Cox-2 in the CNS contributes to inflammatory pain hypersensitivity. Nature. 2001;410(6827):471-475. doi:10.1038/ 35068566

56. Chinery R, Beauchamp RD, Shyr Y, Kirkland SC, Coffey RJ, Morrow JD. Antioxidants reduce cyclooxygenase-2 expression, prostaglandin production, and proliferation in colorectal cancer cells. Cancer Res. 1998;58(11):2323-2327.
57. Laube M, Kniess T, Pietzsch J. Development of antioxidant COX-2 inhibitors as radioprotective agents for radiation therapy-A hypothesis-driven review. Antioxidant. 2016;5(2):14. doi:10.3390/ antiox 5020014

58. Broom DC, Samad TA, Kohno T, Tegeder I, Geisslinger G, Woolf CJ. Cyclooxygenase 2 expression in the spared nerve injury model of neuropathic pain. Neuroscience. 2004;124(4):891-900. doi:10.1016/j.neuroscience.2004.01.003

59. Ma W, Chabot JG, Vercauteren F, Quirion R. Injured nerve-derived COX2/PGE2 contributes to the maintenance of neuropathic pain in aged rats. Neurobiol Aging. 2010;31(7):1227-1237. doi:10.1016/j. neurobiolaging.2008.08.002

60. Jha MK, Jeon S, Suk K. Glia as a link between neuroinflammation and neuropathic pain. Immune Netw. 2012;12(2):41-47. doi:10.4110/ in.2012.12.2.41
International Journal of Nanomedicine

\section{Publish your work in this journal}

The International Journal of Nanomedicine is an international, peerreviewed journal focusing on the application of nanotechnology in diagnostics, therapeutics, and drug delivery systems throughout the biomedical field. This journal is indexed on PubMed Central, MedLine, CAS, SciSearch ${ }^{\mathbb{B}}$, Current Contents ${ }^{\mathbb{B}} /$ Clinical Medicine,

\section{Dovepress}

Journal Citation Reports/Science Edition, EMBase, Scopus and the Elsevier Bibliographic databases. The manuscript management system is completely online and includes a very quick and fair peer-review

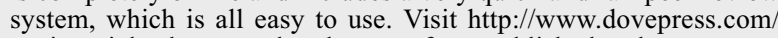
testimonials.php to read real quotes from published authors. 